\title{
Mathematical models of HIV pathogenesis
}

In the May issue of Nature Medicine, Grossman and Herberman ${ }^{1}$ succinctly refute the "tap-and-drain" and "blind homeostasis" models of HIV pathogenesis. Both of these theories attribute a primary role to direct pathogenic mechanisms (either viral cytopathicity or immune clearance of HIVinfected cells). However, the daily loss of infected CD4 cells is maximally estimated to be only $0.05 \%$ of the CD4 cell population, two orders of magnitude lower than normal daily CD4 replenishment - even in the late stages of disease ${ }^{4}$. Elsewhere, a once popular model designed to circumvent this issue (the diversity threshold ${ }^{5}$ ) has not been born out by longitudinal analysis of HIV phenotypes ${ }^{6,7}$.

The reflexive criticism that these mathematical models are "overly simplistic" is not constructive. Mathematical models are intentionally tautological, as they are simply formal statements of theory. The tapand-drain, diversity threshold, and blind homeostasis models make concrete (although not always explicit) assumptions on the relevance and meaning of experimental observations (reflecting their choice of "salient" features). Moreover, they make testable predictions. It is regrettable that these influential theories have not been confirmed; however, non-falsifiable hypotheses have limited scientific value.

Model construction requires assessing the relative importance of many experimental observations. Much of the phenomenology of HIV infection is unaccounted for by the three aforementioned models. Broadly, the issue of "activation" is ignored. Grossman and Herberman make a strong, self-consistent case for a largely "normal" activation becoming increasingly detrimental ("too much of a good thing?"). However, some of the more overtly detrimental effects of HIVinduced activation should not be lightly dismissed. For example, relatively high levels of apoptosis are observed in HIV-infected patients. Since apoptosis occurs predominantly in uninfected CD4 as well as CD8 cells $s^{8}$, it is not readily incorporated into any of the three models. Excessive or dysregulated apoptosis in HIV infection may be related to a proximal pathogenic event, namely, the binding of a virion to the CD4 receptor. It is well documented that CD4 binding (even by non-infectious virions or viral products) can affect many immunological processes, including activation, induction or priming of apoptosis, induc- tion of anergy, clonal deletion, homeostasis and dysregulation.

To construct a formal model, several dynamic relationships need to be empirically quantified. Ideally, such characterization should be unprejudiced by theory, but the intrinsic complexity of the immune system and the limitations of direct observation renders this impossible. Sadly, theories often maintain their influence merely by precedent and authority. Mathematical models are meant to analyze theories, not create them. But mathematical models offer the potential for critical debate over assump. tions and, ultimately, resolution.

Russell W. ANDERSON, PH.D.

Smith-Kettlewell Eye Research Institute 2232 Webster Street

San Francisco, California 94115, USA

email: rwa@milo.berkeley.edu

and

Department of Biomedical Engineering University of Northern California USA Petaluma, California, USA

Grossman and Heberman reply - We agree that direct detrimental effects of HIV should not be lightly dismissed. We note, however, that it is actually the alternative viewpoint, stressing the "normality" of what we consider manifestations of adaptive response? that has not been given sufficient attention. Many of the so called "detrimental effects" can be reinterpreted or may be in-vitro artifacts. While Anderson credits our hypothesis with self-consistency, we would like to note also its increasing consistency with clinical and experimental observations. We have argued that chronic immune activation in itself may account for several immunological changes seen in HIV infection $^{1,8}$. This proposition is supported by recent observations made in a population of Ethiopian immigrants living in Israel (Bentwich \& Grossman, manuscript in preparation). Immunological changes observed in HIV non-infected but immune activated individuals, suffering from helminthic infections, bears a strong resemblance to what has been observed in HIV infected individuals at different stages of the infection. To our knowledge, we were the first to propose T-cell "redistribution" between the tissues and blood, rather than depletion, as a major cause for the observed changes in $\mathrm{T}$ cell counts during the asymp- tomatic phase of HIV infection'. This proposal is supported by recent evidence showing a remarkable discrepancy between these changes in the blood and the numbers and subset ratios of $\mathrm{T}$ cells in the tissues ${ }^{10}$. The scenario of progressive changes, driven by increasing immune istribution, homeostasis and function ${ }^{9}$ has been borne out by recent studies of the long-term effects of drug-combination treatment ${ }^{11}$, in which decreasing the HIV load gradually reversed teh changes in immune parameters. The proposed relationship between activation markers, cellular "anergy" and reduced susceptibility to HIV replication remains to be investigated.

Anderson emphasizes the one virtue of the simple models of HIV pathogenesis, namely, that they are testable. It is a widely-held opinion that a model that makes simple, refutable assumptions can be more valuable than one which is elaborate but difficult to test. This is true only to a point. If a model selects a very limited set of "salient features" on an ad hoc basis, assessment of its value should be deferred until after it is tested; and a model that accounts for a wide range of observations may deserve an extra effort to test or refute.

Anderson also reminds us that the role of mathematical models is only to provide formal statements of theory. Their formulation and analysis should facilitate debate over assumptions. In reality, mathematical models, rather that illuminating the picture, can be misleading. It is all too easy to present a biased set of assumptions as a "natural" choice and the product of reasonable simplifications. A modeler's bias may be shared by a majority of the readers but many may not be well qualified to spot tacit assumptions that affected the results.

Certainly mathematical models offer the potential for critical debate. It is interesting, considering the spectacular rise of the "new view of HIV" any visible debate over this versus alternative theories, especially in the light of new findings ${ }^{13,14}$. We also note that the once very popular and attractive theories have been fading away rather silently.

\section{Zvi GROSSMAN ${ }^{1} \&$}

RONALD B. HERBERMAN ${ }^{2}$

'Dept. of Physiology and Pharmacology

Sackler Facility of Medicine, Tel Aviv University

Tel Aviv 69978, Israel

${ }^{2}$ Univ. of Pittsburgh Cancer Institute

Pittsburgh, Pennsylvania 15213-3221, USA 